Apidologie, 1986, 17 (3), 217-226

\title{
COMPARISON OF AFRICANIZED AND EUROPEAN QUEEN-MATING COLONIES IN VENEZUELA
}

\author{
Richard L. HELLMICH II, Robert G. DANKA, Thomas E. RINDERER, \\ and Anita M. COLLINS \\ U.S.D.A., A.R.S., Honey-Bee Breeding, Genetics and Physiology Laboratory \\ 1157 Ben Hur Road, Baton Rouge, Louisiana 70820, U.S.A.
}

\section{SUMMARY}

Africanized queen-mating colonies produced fewer mated queens $(\mathbf{P}<0.05)$, absconded more $(\mathrm{P}<0.001)$ and had more population dwindling $(\mathrm{P}<0.03)$ when compared to European queenmating colonies. Africanized colonies most affected by these factors were 5-1 nuclei, the type of mating nuclei most commonly used by U.S. queen producers. Efficiency of queen production was increased by doubling bee populations and hive volume and by adding brood.

\section{INTRODUCTION}

Small-sized mating colonies have been the cornerstone of American queen production since Henry Alley began using them extensively nearly a century ago. The double-chambered small nucleus and the «baby» nucleus are the most common units used by queen producers today because they are the most economical way to produce queens on a large scale. However, small colonies may be an invitation to hardship when the population of Africanized bees spread as far north as the United States ${ }^{(1)}$.

The Committee on the African Honey Bee (Michener, 1972) after interviewing several bee scientists and beekeepers in South America, concluded that queen mating with Africanized bees in small nucleus colonies would be difficult or impossible. Even in South Africa, native territory of the African bee (A. $m$. scutellata), high rate of queen loss due to absconding, swarming and

(1) Africanized bees are decendants of Apis mellifera scutellata (formerly called adansonii) imports and their hybrids with various subspecies previously imported to South America. 
supersedure has made requeening and thus queen production impractical (Fletcher and Tribe, 1977).

The precise nature of the Africanized bee challenge for U.S. beekeepers will not be known until they arrive in the southern U.S. The projected time of arrival is 1988-1990. Nevertheless, predictions for the U.S. can be made based on research performed on the Africanized bee in Venezuela. Objectives of this study were to : 1) determine if Africanized mating nuclei would be practical for queen production and 2) determine the importance of space, bee population and brood for increasing efficiency of Africanized mating nuclei.

\section{MATERIALS AND METHODS}

The study site was near Sarare, Venezuela, at the edge of a tropical forest. A small stream, located within 20 meters of the mating yard, provided a source of water, and trees provided ample shade.

Matings units were small double-chambered hives (five liters per chamber) similar to nuclei commonly used by commercial queen producers. Each chamber contained two frames with combs measuring $19 \times 13 \mathrm{~cm}$ and a division-board feeder with a $500 \mathrm{ml}$ capacity. These feeders, one in the 5-1 nuclei and two in the 10-1 nuclei, were kept full of sugar syrup throughout the experiment. The 10-1 unit was made by removing a partition board which normally separates the two chambers of these nuclei. Units were placed on metal stands which had each leg immersed in oil to protect colonies from predators (primarily Camponotus spp. of ants).

Three treatments were used : bee type (Africanized and European), nucleus size (5-1 and 10-1) and brood status (brood and no brood). Brood (150-250 sq. cm) was obtained from Africanized and European colonies (2). Eggs and young larvae were used to ensure brood did not emerge before the first two inspections. Workers were shaken into screen containers $(51 \times 41 \times 41 \mathrm{~cm})$ from established Africanized and European colonies. Bees in these containers were fed sugar syrup and kept in storage $\left(21^{\circ} \mathrm{C} \pm 1{ }^{\circ} \mathrm{C}\right)$. At dusk, two to three days later, a virgin queen (less than 24 hours old) from an Africanized source was caged on a comb (hardware-cloth push-in cage) and put into each mating unit. Measured quantities of bees were then added to each hive (approximately $227 \mathrm{~g}$ for the 5-1 units and $454 \mathrm{~g}$ for the 10-1 units).

Bees were confined in the hives for two days then released in the evening. Inspections were made 4, 11 and 21 days after bees were released. Virgin queens were released during the first inspection. During the first and second inspections, bee population (estimated to the nearest tenth of a comb of bees) and presence of queen were observed for each colony. Presence or absence of a laying queen was noted for each colony during the third inspection. The mating was called "successful» when the queen was laying and the colony had seven-tenths of a frame of bees, which was considered sufficient bee population for another mating rotation. Whether a colony absconded was also noted during the inspections. Fletcher (1975) stated that absconding occurs when a colony abandons a nestsite and establishes itself at another location. Even though in most instances establishment at a different location could not be verified, any abandoned hive was considered to be the result of absconding.

Two trials, each with 56 colonies (seven replicates per combination of treatments) were conducted. Bees for the first trial were shaken March 30 and bees for the second trial were shaken April 4, 1984.

(2) European queens were purchased from commercial producers in the U.S. Africanized queens were from swarms caught locally in Venezuela. 
Weather was typical of the Venezuelan dry season. Temperature ranged from $24-37^{\circ} \mathrm{C}$ and relative humidity varied from $30-80 \%$.

Chi-Square $2 \times 2$ tests of independence were used to analyze bee-type, nucleus-size, broodstatus and trial effects for mating-success and absconding data. Specific comparisons were made that allowed us to compare bee types in 5-1 units (the standard unit for queen producers) and to determine whether increasing colony size or adding brood increased efficiency of Africanized units. Trial comparisons of the Africanized colonies were made to check for production consistency. Analysis of variance ( 24 factorial design) was used to analyze bee-population data. Bee type, nucleus size, brood and trial were used as grouping factors.

\section{RESULTS}

\section{Mating Success and Absconding}

More queens were mated successfully in European colonies than in Africanized colonies $(\mathrm{P}<0.05)$, and in colonies with brood than in colonies without brood $(\mathrm{P}<0.05)$ (Tabl. 1). The 5-1 European colonies produced more queens

TABL. 1. - Number of colonies with successfully mated queens

for seven replicates of 16 bee-type [Africanized (A), European (E)] nucleus size (5-l, 10-l), brood-status [no brood (No B.), brood (B period)], and trial [trial 1 ( $T$ 1), trial 2 (T2)] combinations, and $2 \times 2$ tables for main effects and planned comparisons with $\chi_{2}$ Probabilities period.

\begin{tabular}{|c|c|c|c|c|c|}
\hline & & \multicolumn{4}{|c|}{ Mating successes } \\
\hline & & \multicolumn{2}{|c|}{$5-1$ nucleus } & \multicolumn{2}{|c|}{ 10-1 nucleus } \\
\hline & & trial 1 & trial 2 & trial 1 & trial 2 \\
\hline \multirow[t]{2}{*}{ No brood } & A & 3 & 0 & 3 & 2 \\
\hline & $\mathrm{E}$ & 2 & 4 & 4 & 3 \\
\hline \multirow[t]{2}{*}{ Brood } & A & 3 & 2 & 5 & 3 \\
\hline & $\mathrm{E}$ & 4 & 5 & 6 & 4 \\
\hline
\end{tabular}

Main effects

Successes

Failures

\begin{tabular}{|c|c|c|c|c|c|c|c} 
A & E & B. & No B. & $5-1$ & $10-1$ & T 1 & T 2 \\
\hline 21 & 32 & 32 & 21 & 23 & 30 & 30 & 23 \\
35 & 24 & 24 & 35 & 33 & 26 & 26 & 33 \\
$(\mathrm{p}<0.05)$ & $(\mathrm{p}<0.05)$ & \multicolumn{2}{|c|}{ N.S. } & \multicolumn{2}{|c}{ N.S. }
\end{tabular}

Planned comparisons

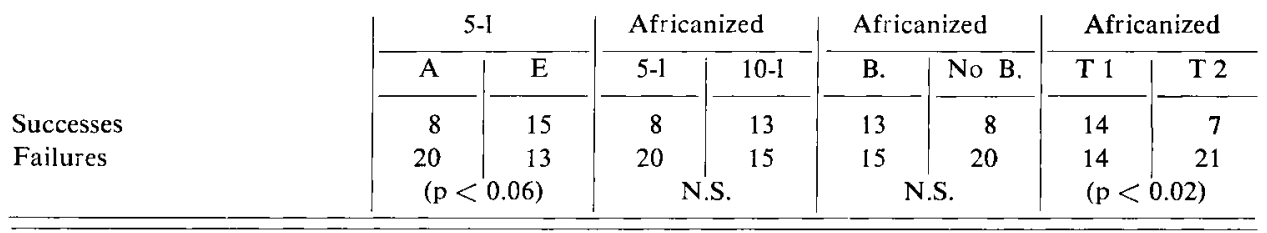


than 5-1 Africanized colonies $(\mathrm{P}<0.06)$. Neither increasing colony size nor adding brood appreciably increased production of mated queens in Africanized units. Fewer Africanized queens were produced in Trial 2 than in Trial 1 $(\mathrm{P}<0.02)$.

More Africanized colonies absconded than European colonies $(P<0.001)$, and more colonies absconded from 5-1 units than 10-1 units $(\mathrm{P}<0.02)$ (Tabl. 2). Fewer colonies absconded during Trial 1 than Trial $2(\mathrm{P}<0.02)$. Absconding from 5-1 Africanized units was higher than absconding from 5-1 European units $(\mathrm{P}<0.001)$ and 10-1 Africanized units $(\mathrm{P}<0.01)$. Fewer Africanized units absconded during Trial 1 than Trial $2(\mathrm{P}<0.02)$.

TABL. 2. - Number of colonies which absconded for seven replicates of 16 bee-type, nucleus-size, brood-status and trial combinations, and $2 \times 2$ tables for main effects and planned comparisons with $x^{2}$ Probabilities. Treatments are designated as in Table 1 period

\begin{tabular}{|c|c|c|c|c|c|}
\hline & & \multicolumn{4}{|c|}{ Abscondings } \\
\hline & & \multicolumn{2}{|c|}{$5-1$ nucleus } & \multicolumn{2}{|c|}{ 10-1 nucleus } \\
\hline & & trial 1 & trial 2 & trial 1 & trial 2 \\
\hline \multirow[t]{2}{*}{ No brood } & $\mathbf{A}$ & 2 & 6 & 0 & 1 \\
\hline & $\mathbf{E}$ & 0 & 0 & 0 & 0 \\
\hline \multirow[t]{2}{*}{ Brood } & A & 1 & 3 & 0 & 1 \\
\hline & $\mathbf{E}$ & 0 & 0 & 0 & 1 \\
\hline
\end{tabular}

Absconded Remained

\begin{tabular}{|c|c|c|c|c|c|c|c|}
\hline \multicolumn{8}{|c|}{ Main effects } \\
\hline A & $E$ & B. & No $B$. & $5-1$ & $10-1$ & T 1 & T 2 \\
\hline 14 & 1 & 6 & 9 & 12 & 3 & 3 & 12 \\
\hline 42 & 55 & 50 & 47 & 44 & 53 & 53 & 44 \\
\hline \multicolumn{2}{|c|}{$(\mathrm{p}<0.001)$} & \multicolumn{2}{|c|}{ N.S. } & \multicolumn{2}{|c|}{$(\mathrm{p}<0.02)$} & \multicolumn{2}{|c|}{$(p<0.02)$} \\
\hline
\end{tabular}

Planned comparisons

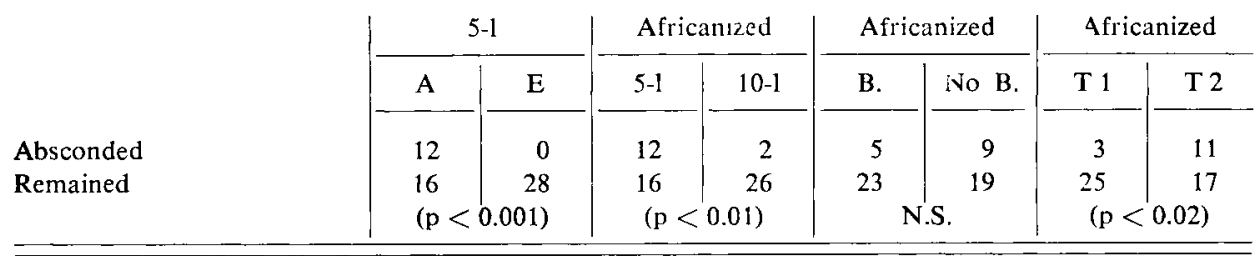

The percentage of colonies that absconded from remaining mating units was calculated on a per-day basis for periods preceeding first, second and third 
inspections (Tabl. 3). In each case, when absconding occurred percentages were higher for Africanized colonies than for European colonies. Absconding rates were higher for the periods preceeding the second (11 days after release) and third (21 days after release) inspections than the period preceeding the first inspection (4 days after release).

TABL. 3. - Mean daily percent of colonies to abscond from remaining $A$ fricanized $(A)$ and European $(E)$ mating units for a four-day period preceeding the first inspection, a seven-day period preceeding the second inspection and a ten-day period preceeding the third inspection

\begin{tabular}{c|r|r|r|r|r}
\hline \multirow{2}{*}{ Inspection } & Days after bees released & \multicolumn{2}{|c}{ Mean daily percentage of colonies absconded } \\
\cline { 3 - 5 } & & Trial 1 & \multicolumn{2}{|c}{ Trial 2 } \\
\hline & 4 & $\mathrm{~A}$ & $\mathrm{E}$ & $\mathrm{A}$ & $\mathrm{E}$ \\
\hline 1 & 11 & 0 & 0 & 2.7 & 0 \\
2 & 21 & 1.5 & 0 & 4.6 & 0.5 \\
3 & 1.6 & 1.4 & 5.3 & 3.6 \\
\hline
\end{tabular}

\section{Bee Populations}

Nucleus colonies stocked with European bees had larger bee populations than those stocked with Africanized bees at the first inspection (mean \pm std. error : $\left.\overline{\mathrm{X}}_{\mathrm{E}}=2.65 \pm 0.11, \overline{\mathrm{X}}_{\mathrm{A}}=1.77 \pm 0.08\right)(\mathrm{P}<0.0001)$ and at the second inspection $\left(\overline{\mathrm{X}}_{\mathrm{E}}=1.76 \pm 0.11, \overline{\mathrm{X}}_{\mathrm{A}}=1.50 \pm 0.09\right)(\mathrm{P}<0.03)$. Differences between bee types are apparent in the population means for the nucleus-size, brood-status and trial combinations (Tabl. 4). In nearly every case, European nuclei had greater populations than Africanized nuclei.

Africanized bee populations also varied more from trial to trial than European bee populations. For example, first inspection data indicate Africanized bee populations were $36 \%$ lower during the second trial $(1.37 \pm 0.11$ combs of bees) than they were during the first trial $(2.14 \pm 0.11$ combs of bees). However, European bee populations were only $10 \%$ lower during the second trial $(2.51 \pm 0.11$ combs of bees) than they were during the first trial $(2.79 \pm 0.11$ combs of bees). This bee-type by trial interaction is significant for the first inspection $(\mathrm{P}<0.03)$.

Colonies that were given brood had larger bee populations than colonies that were not given brood at both the first inspection $\left(\overline{\mathrm{X}}_{\mathrm{broodl}}=2.35 \pm 0.08\right.$, $\left.\mathrm{X}_{\text {no brood }}=2.08 \pm 0.08\right)(P<0.0001)$ and second inspection $\left(\mathrm{X}_{\text {brood }}=1.88 \pm 0.12\right.$, 
$\left.\overline{\mathrm{X}}_{\mathrm{nobrwa}}=1.40 \pm 0.11\right)(\mathrm{P}<0.005)$. The $5-1$ nuclei had smaller bee populations than 10-1 nuclei also at both the first inspection $\left(\overline{\mathrm{X}}_{5-1}=1.78 \pm 0.08, \overline{\mathrm{X}}_{10)-1}=\right.$ $2.66 \pm 0.08)(\mathrm{P}<0.0001)$ and second inspection $\left(\bar{X}_{51-}=1.34 \pm 0.12, \bar{X}_{10-1}=\right.$ $1.91 \pm 0.11)(\mathrm{P}<0.0007)$.

TABL. 4. - Mean combs of bees $(19 \times 13 \mathrm{~cm})$ for inspections 1 and 2, and nucleus-size, brood-status and trial combinations of Africanized (A) and European (E) mating colonies period. Absconded colonies are not included. (Mean square error : Inspection $1=0.336$, Inspection $2=0.662$ )

\begin{tabular}{|c|c|c|c|c|c|c|c|}
\hline \multirow{2}{*}{ Inspection } & \multirow{2}{*}{$\begin{array}{c}\text { Nucleus } \\
\text { size }\end{array}$} & \multirow{2}{*}{ Brood } & \multirow{2}{*}{$\begin{array}{l}\text { type } \\
\text { Bee }\end{array}$} & \multicolumn{2}{|c|}{ Trial 1} & \multicolumn{2}{|c|}{ Trial 2} \\
\hline & & & & n & Bees & $\mathrm{n}$ & Bees \\
\hline \multirow[t]{8}{*}{1} & \multirow[t]{4}{*}{$5-1$} & \multirow[t]{2}{*}{ No } & A & 7 & 1.40 & 6 & 0.87 \\
\hline & & & $\mathrm{E}$ & 7 & 1.90 & 7 & 1.79 \\
\hline & & \multirow[t]{2}{*}{ Yes } & A & 7 & 1.87 & 7 & 1.49 \\
\hline & & & $\mathrm{E}$ & 7 & 2.60 & 7 & 2.17 \\
\hline & \multirow[t]{4}{*}{$10-1$} & \multirow[t]{2}{*}{ No } & A & 7 & 2.43 & 5 & 1.80 \\
\hline & & & $\mathrm{E}$ & 7 & 3.21 & 7 & 2.99 \\
\hline & & \multirow[t]{2}{*}{ Yes } & A & 7 & 2.87 & 7 & 1.38 \\
\hline & & & $\mathrm{E}$ & 7 & 3.44 & 7 & 3.10 \\
\hline \multirow[t]{8}{*}{2} & \multirow[t]{4}{*}{$5-1$} & \multirow[t]{2}{*}{ No } & A & 5 & 1.06 & 1 & 0.30 \\
\hline & & & $\mathrm{E}$ & 7 & 1.03 & 7 & 0.94 \\
\hline & & \multirow[t]{2}{*}{ Yes } & A & 6 & 1.45 & 4 & 1.05 \\
\hline & & & E & 7 & 2.04 & 7 & 1.74 \\
\hline & \multirow[t]{4}{*}{$10-1$} & \multirow[t]{2}{*}{ No } & A & 7 & 1.97 & 5 & 1.12 \\
\hline & & & $\mathrm{E}$ & 7 & 2.64 & 7 & 0.99 \\
\hline & & \multirow[t]{2}{*}{ Yes } & A & 7 & 2.52 & 7 & 1.07 \\
\hline & & & E & 7 & 2.91 & 6 & 1.80 \\
\hline
\end{tabular}

\section{DISCUSSION}

Many activities of the Africanized honey bee have been cited as undesirable for commercial apiculture. Most significant may be their defensive behavior (Collins et al., 1982), which can lead to dangerous stinging encounters with beekeepers and the general public. However, our study suggests that poor mating success, absconding, and population dwindling may be equally important to queen producers. 
Africanized mating colonies absconded more then twelve times more than European mating colonies ( $25 \%$ vs. $2 \%$ of total colonies), and had approximately one-third fewer mating successes and lost one-third more stocked worker bees after 21 days. Single-chambered Africanized colonies were particularly affected by these factors - $43 \%$ absconded (compared with $0 \%$ Europeans) and only $29 \%$ had successful matings (compared with $54 \%$ Europeans).

Winston et al. (1979) noted several probable causes of absconding within two basic categories : disturbance induced (by beekeeper manipulations, predators, inferior nesting sites) and seasonally induced (by rainfall, temperature extremes, resource dearths). Beekeeper manipulations and the inferior nest of the small hive were probably the most important factors in this experiment. Either of these two factors could have produced environments which were more stressful to Africanized colonies than European colonies.

Evidence that Africanized bees are more easily disturbed than European bees by changes in the environment is suggested by colony performances in the second trial. Differences between the two bee types were greater during the second trial than they were during the first trial. Many factors could account for these trial differences, for example, variable weather conditions, slight changes in colony management or slight differences in genetic stock. However, these trial differences suggest queen-mating success with Africanized mating nuclei may not be as reliable as it is with European mating nuclei. Such variability has important practical implications because queen producers must have a reliable source of queens.

Efficiency of queen production can be increased by doubling bee population and volume of the hive, and also by adding brood. VogEL (1985), has had limited success mating queens in large (32 l) Africanized mating colonies. These mating colonies are approximately six times larger than the 5-1 nucleus so they do not represent an economical solution. Nevertheless, these observations suggest queen mating with Africanized colonies can be done if one is willing to use a larger colony.

Numbers of successful matings by queens from European colonies (overall, $57 \%$ ) were not high by most queen-breeding standards. Comb manipulations required to make inspections, low drone populations prior to the rainy season and honey-bee predators (insects and birds) could all have contribued to reduced mating success.

This study substantiates the concerns of the Committee on the African Bee. Large-scale queen mating with Africanized mating nuclei will be difficult and perhaps impossible with established queen-production technology. In order to produce queens economically queen producers must use small mating units and intensively manipulate them. When Africanized bees are used to populate mating 


\section{hives both of these factors appear to contribute to reduced mating success, absconding, and population dwindling.}

Received for publication in June 1985. Accepted for publication in May 1986.

\section{ACKNOWLEDGEMENTS}

The experiment was conducted in cooperation with the Louisiana Agricultural Experiment Station and Universidad Centro Occidental Lisandro Alvarado, Barquisimeto, Venezuela. The Authors wish to thank Alcides Escalona, Roberto Colmenares, J. Anthony Stelzer and Vicki LANCASTER for technical assistance.

\section{RESUME \\ COMPARAISON DE COLONIES DE FECONDATION D'ABEILLES AFRICANISEES ET EUROPEENNES AU VENEZUELA}

Les objectifs de cette étude sont : 1) déterminer si des nuclei de fécondation avec des abeilles africanisées seraient pratiques pour produire des reines;2) déterminer l'èp̈pace nécessaire, la taille de la population d'abeilles et de couvain pour accroître l'efficacité des nuclei de fécondation d'abeilles africanisées.

Les unités de fécondation sont de petites ruches à 2 corps ( 5 litres par corps). Chaque corps contient 2 cadres avec un rayon de $19 \times 13 \mathrm{~cm}$ et un nourrisseur d'une capacité de $500 \mathrm{ml}$. L'unité de 101 est obtenue en ôtant la planche qui sépare normalement les 2 corps de ces nuclei.

On a secoué dans des récipients grillagés des abeilles d'origine africanisée ou européenne sûre. Au crépuscule 2 ou 3 jours plus tard, on a placé sur un rayon une cage à reine avec une reine vierge africanisée (âgée de moins de $24 \mathrm{~h}$ ) dans chaque unité de fécondation. On a ensuite ajouté à chaque ruche des quantités connues d'abeilles (environ $227 \mathrm{~g}$ pour les unités de 51 et $454 \mathrm{~g}$ pour les unités de $10 \mathrm{l}$ ). Les abeilles ont été confinées dans la ruche pendant 2 jours, puis lâchées dans la soirée. Les reines vierges ont été libérées au cours de la première visite.

On a appliqué 3 traitements portant sur : a) le type d'abeilles (africanisées ou européennes); b) la taille du nucleus (5 l et $10 \mathrm{I}$ ) et c) la présence ou l'absence de couvain. Deux essais ont été faits, chacun sur 56 colonies (7 répétitions par combinaison de traitements).

Les colonies de fécondation africanisées ont déserté plus de 12 fois plus que les colonies de fécondation européennes ( $25 \%$ contre $2 \%$ de l'ensemble des colonies) et ont eu environ un tiers de moins de réussites dans les fécondations et perdu un tiers de plus d'ouvrières dans les 21 jours. Les colonies africanisées à un corps ont été particulièrement touchées par ces facteurs : elles ont déserté à $43 \%$ (contre $0 \%$ pour les européennes) et n'ont eu que $29 \%$ de réussites dans les fécondations (contre $54 \%$ pour les européennes).

Cette étude montre donc que l'efficacité de la production de reines peut être accrue en doublant la population d'abeilles et le volume de la ruche et aussi en ajoutant du couvain. 


\section{ZUSAMMENFASSUNG}

\section{VERGLEICH AFRIKANISIERTER UND EUROPAISCHER BEGATTUNGSVÖLKCHEN IN VENEZUELA}

\section{Das Ziel dieser Untersuchung war :}

1.) Feststellung der Eignung von Begattungsvölkchen mit Afrikanisierten Bienen (Hybriden von Apis mellifera scutellata) für die praktische Königinnenzucht.

2.) Bestimmung der Bedeutung von Kastengröße, Volksstärke und der Zugabe von Brut für eine Steigerung der Ergebnisse mit Afrikanisierten Begattungsvölkchen.

Die Begattungseinheiten waren kleine Beuten mit zwei Abteilen (5 Liter je Abteil). Jedes Abteil enthielt zwei Rähmchen mit Waben von der Größe $19 \times 13 \mathrm{~cm}$ und eine Futtertasche mit $500 \mathrm{ml}$ Inhalt. Die 10-Liter-Einheit wurde durch Entfernung des Schiedbrettes erzielt, das sonst die beiden Abteile dieser Begattungskästen trennt.

Afrikanisierte und europäische Bienen aus Völkern, die als Afrikanisiert oder europäisch bekannt waren, wurden getrennt in vergitterte Kisten abgeklopft. Zwei bis drei Tage später wurde in der Dämmerungszeit eine Jungkönigin (unter $24 \mathrm{~h}$ alt) auf einer Wabe gekäfigt in jedes Abteil gesetzt. $\mathrm{Zu}$ jedem Kasten wurde eine abgemessene Menge Bienen (ungefähr $227 \mathrm{~g}$ für die 5 LiterEinheiten und $454 \mathrm{~g}$ für die 10 Liter-Einheiten) zugegeben. Die Völkchen wurden für zwei Tage in den Kästen gesperrt gehalten und dann am Abend freigegeben. Inspektionen wurden 4, 11 und 21 Tage nach Freigabe des Fluges durchgeführt. Bei der ersten Inspektion wurden die Königinnen freigegeben.

Es wurden drei Versuchsansätze durchgeführt - Art der Bienen (Afrikanisiert und europäisch), Kastengröße (5 Liter und 10 Liter) und Brut (Brut und keine Brut). Der Versuch wurde zweimal angesetzt, jedes Mal mit 56 Völkchen.

Afrikanisierte Völkchen schwärmten vierzehn Mal so häufig ab als europäische Begattungsvölkchen $(25,0 \%$ gegen $1,8 \%)$, sie hatten einen um ein Drittel geringeren Begattungserfolg und wiesen nach 21 Tagen einen um ein Drittel höheren Abgang an Bienen auf. Afrikanisierte Völkchen in Einzelabteilen waren von diesen Faktoren besonders betroffen - 42,9\% zogen aus (verglichen mit $0 \%$ europäischen) und nur $28,6 \%$ wurden erfolgreich gepaart (gegen $53,6 \%$ europäische Völkchen).

Diese Untersuchung zeigt überdies, daß das Ergebnis der Königinnenzucht mit Afrikanisierten Bienen durch Verdoppelung der Volksstärke und der Kastengröße, sowie durch Zugabe von Brut verbessert werden kann.

Die Behandlung der Afrikanisierten Begattungsvölkchen wurde durch ihr Verteidigungsverhalten nicht behindert. Diese kleinen Völkchen mit Afrikanisierten Bienen zeigten keine besondere Verteidigungstendenz.

\section{REFERENCES}

Collins A.M., Rinderer T.E., Harbo J.R., Bolten A.B., 1982. - Colony defense by Africanized and European honey bees. Science, 218, 72-74.

Fletcher D.J.C., 1975. - New perspectives in the causes of absconding in the African bee (Apis mellifera adansonii L.), Part I, S. Afr. Bee J., 47, 11-14. 
Fletcher D.J.C., Tribe G.D., 1977. - Natural emergency queen rearing by the African bee A. $m$. adansonii and its relevance for successful queen production by beekeepers, I. Proceedings Apimondia International Symposium, Pretoria, South Africa. Nov. 17-19, 1976, 132-140.

Michener C.D. (Chairman), 1972. - Committee on the African honey bee, National Research Council, Agricultural Research Service, National Technical Information Service, U.S. Department of Commerce.

Vogel W., 1985 (personal communication). - General manager of Miel Primavera, Apdo. 186, Acarigua, Portuguesa, Venezuela 3301A.

Winston M.L., Otis G.W., TAYlor O.R. Jr., 1979 - Absconding behaviour of the Africanized honeybee in South America. J. Apic. Res.. 18, 85-94. 